\title{
Weighted Integrals of Polynomial Splines
}

\author{
Mladen Rogina \\ Department of Mathematics, University of Zagreb, 10002 Zagreb, Croatia, \\ rogina@math.hr
}

Summary. The construction of weighted splines by knot insertion techniques such as de Boor and Oslo - type algorithms leads immediately to the problem of evaluating integrals of polynomial splines with respect to the positive measure possessing piecewise constant density. It is for such purposes that we consider one possible way for simple and fast evaluation of primitives of products of a polynomial B-spline and a positive piecewise constant function.

\section{Introduction and Motivation}

Weighted splines appear in many applications, the most well-known being the cubic version where they arise naturally in minimizing functionals like $V(f):=$ $\sum_{i=1}^{n}\left(w_{i} \int_{t_{i}}^{t_{i+1}}\left[D^{2} f(t)\right]^{2} d t, w_{i}>0\right.$, sometimes also accompanied by the control of first derivatives: $V(f):=\sum_{i=1}^{n}\left(w_{i} \int_{t_{i}}^{t_{i+1}}\left[D^{2} f(t)\right]^{2} d t+\nu_{i} \int_{t_{i}}^{t_{i+1}}[D f(t)]^{2} d t\right), \nu_{i} \geq 0$, $w_{i}>0$, see $[6,7,9]$ and [11] for a bivariate version.

The parametric version is often used as a polynomial alternative to the exponential tension spline in computer-aided geometric design, and some shape-preserving software systems (MONCON, TRANSPLINE) have been written for that purpose $[13,9,10]$. It is known that the associated B-splines can be calculated by the knot insertion algorithms. For the cubic version of weighted splines, explicit expressions for the knot insertion matrices exist, which are of the very simple form $[8,14]$. In the case of the knot insertion algorithms can in principle be obtained by specializing the general theory of Chebyshev blossoming [12].

Weighted splines can also be evaluated by an integrated version of the derivative formula [15], which can also be used to define most general Chebyshev B-splines [1]:

$$
B_{i, d \boldsymbol{\sigma}}^{n}(x)=\frac{1}{C_{n-1}(i)} \int_{t_{i}}^{x} B_{i, d \boldsymbol{\sigma}^{(1)}}^{n-1} d \sigma_{2}-\frac{1}{C_{n-1}(i+1)} \int_{t_{i+1}}^{x} B_{i+1, d \boldsymbol{\sigma}^{(1)}}^{n-1} d \sigma_{2}
$$

where $B_{i, d \boldsymbol{\sigma}}^{n}(x)$ is the $n^{\text {th }}$-order Chebyshev spline, $d \boldsymbol{\sigma}=\left(d \sigma_{2} \ldots d \sigma_{n}\right)^{T}$ is the measure vector and $d \boldsymbol{\sigma}^{(1)}=\left(d \sigma_{3} \ldots d \sigma_{n}\right)^{T}$ is the measure vector with respect to the first reduced system. We assume that $d \sigma_{i}$ are some Stieltjes measures, and that all 
the B-splines in question are normalized so as to make a partition of unity. The constants in the denominators are integrals of B-splines over its support, with respect to the measure that is missing in the definition of $d \boldsymbol{\sigma}^{(1)}$ :

$$
C_{n-1}(i):=\int_{t_{i}}^{t_{i+n-1}} B_{i, d \sigma^{(1)}}^{n-1} d \sigma_{2} .
$$

The numerical stability of (1) is doubtful (even for polynomial splines), so evaluation by knot insertion is preferred. However, for weighted splines we need only very simple measures, which are all but one Lebesgue measures, and the one that is not has density which is piecewise constant and positive. To be more precise, weighted B-splines are piecewisely spanned by the Chebyshev system of weighted powers:

$$
\begin{aligned}
u_{1}(x) & =1 \\
u_{2}(x) & =\int_{a}^{x} d \tau_{2} \\
u_{3}(x) & =\int_{a}^{x} d \tau_{2} \int_{a}^{\tau_{2}} \frac{d \tau_{3}}{w\left(\tau_{3}\right)} \\
& \vdots \\
u_{k}(x) & =\int_{a}^{x} d \tau_{2} \int_{a}^{\tau_{2}} \frac{d \tau_{3}}{w\left(\tau_{3}\right)} \int_{a}^{\tau_{3}} d \tau_{4} \cdots \int_{a}^{\tau_{k-1}} d \tau_{k} .
\end{aligned}
$$

Finally, one can use algorithms for ordinary polynomial splines and avoid explicit mentioning of weighted splines, but even then integration of products of polynomial splines and piecewise constant function must be performed, as shown by de Boor [3], who also gives closed formulæ for some lower order splines.

\section{Recurrence for Integrals of Polynomial B-Splines}

Whatever approach we choose, in order to evaluate weighted splines we need to calculate the integrals of ordinary polynomial B-splines

$$
C_{k}(j)=\int_{t_{j}}^{t_{j+k}} B_{j}^{k}(\tau) \frac{d \tau}{w(\tau)} .
$$

In what follows, we assume that $B_{j}^{k}$ are normalized so as to make the partition of unity, and that the knot sequence $\left\{t_{j}\right\}$, possibly containing multiple knots, coincides with the breakpoint sequence for $w$. For notation purposes, let $\left.w\right|_{\left[t_{i}, t_{i+1}\right)}=w_{i}$ which makes $w$ right-continuous. We want to find a recurrence for primitives of polynomial B-splines with respect to the piecewise constant positive function $w$, i.e.,

$$
\int_{t_{i}}^{x} B_{i}^{k}(\tau) \frac{d \tau}{w(\tau)}, \quad x \in\left[t_{i}, t_{i+k}\right],
$$

and, specially:

$$
\int_{t_{j}}^{t_{j+1}} B_{i}^{k}(\tau) \frac{d \tau}{w(\tau)}, \quad j=i, \ldots, i+k-1 .
$$

Let $x \in\left[t_{j}, t_{j+1}\right)$, then 


$$
\begin{aligned}
\int_{t_{i}}^{x} B_{i}^{k}(\tau) \frac{d \tau}{w(\tau)}= & \sum_{s=i}^{j-1} \int_{t_{s}}^{t_{s}+1} B_{i}^{k}(\tau) \frac{1}{w_{s}} d \tau+\frac{1}{w_{j}} \int_{t_{j}}^{x} B_{i}^{k}(\tau) d \tau \\
= & \sum_{s=i}^{j-1} \frac{1}{w_{s}}\left(\int_{t_{i}}^{t_{s}+1} B_{i}^{k}(\tau) d \tau-\int_{t_{i}}^{t_{s}} B_{i}^{k}(\tau) d \tau\right) \\
& +\frac{1}{w_{j}}\left(\int_{t_{i}}^{x} B_{i}^{k}(\tau) d \tau-\int_{t_{i}}^{t_{j}} B_{i}^{k}(\tau) d \tau\right) \\
= & \sum_{s=i}^{j-1} \frac{1}{w_{s}} \frac{t_{i+k}-t_{i}}{k}\left(\sum_{r=i}^{s} B_{r}^{k+1}\left(t_{s+1}\right)-\sum_{r=i}^{s-1} B_{r}^{k+1}\left(t_{s}\right)\right) \\
& +\frac{1}{w_{j}} \frac{t_{i+k}-t_{i}}{k}\left(\sum_{r=i}^{j} B_{r}^{k+1}(x)-\sum_{r=i}^{j-1} B_{r}^{k+1}\left(t_{j}\right)\right),
\end{aligned}
$$

by the well known formula for integrals of polynomial splines [16, p. 200] and [2, pp. 150-151]. Let

$$
\bar{\alpha}_{i, j+1}^{k+1}(x):=\sum_{r=i}^{j} B_{r}^{k+1}(x) \quad \text { and } \quad \alpha_{i, j+1}^{k+1}:=\bar{\alpha}_{i, j+1}^{k+1}\left(t_{j+1}\right) .
$$

Then in terms of $\bar{\alpha}$ 's formula (2) can be written as

$$
\int_{t_{i}}^{x} B_{i}^{k}(\tau) \frac{d \tau}{w(\tau)}=\frac{t_{i+k}-t_{i}}{k}\left(\sum_{s=i}^{j-1} \frac{1}{w_{s}}\left(\alpha_{i, s+1}^{k+1}-\alpha_{i, s}^{k+1}\right)+\frac{1}{w_{j}}\left(\bar{\alpha}_{i, j+1}^{k+1}(x)-\alpha_{i, j}^{k+1}\right)\right) .
$$

We claim that $\bar{\alpha}_{i, j+1}^{k+1}(x)$ can be evaluated as convex combination of lower order quantities $\bar{\alpha}_{i, j}^{k}(x)$. By de Boor-Cox recurrence

$$
\begin{aligned}
& \sum_{r=i}^{j} B_{r}^{k+1}(x)=\sum_{r=i}^{j}\left(\frac{x-t_{r}}{t_{r+k}-t_{r}} B_{r}^{k}(x)+\frac{t_{r+k+1}-x}{t_{r+k+1}-t_{r+1}} B_{r+1}^{k}(x)\right) \\
& =\sum_{r=i}^{j} \frac{x-t_{r}}{t_{r+k}-t_{r}} B_{r}^{k}(x)+\sum_{r=i}^{j} B_{r+1}^{k}(x)-\sum_{r=i}^{j} \frac{x-t_{r+1}}{t_{r+k+1}-t_{r+1}} B_{r+1}^{k}(x) \\
& =\sum_{r=i+1}^{j}\left(\frac{x-t_{r}}{t_{r+k}-t_{r}}-\frac{x-t_{r}}{t_{r+k}-t_{r}}\right) B_{r}^{k}(x)+\frac{x-t_{i}}{t_{i+k}-t_{i}} B_{i}^{k}(x)+\sum_{r=i}^{j-1} B_{r+1}^{k}(x) \\
& =\frac{x-t_{i}}{t_{i+k}-t_{i}} B_{i}^{k}(x)+\sum_{r=i+1}^{j} B_{r}^{k}(x)=\frac{x-t_{i}}{t_{i+k}-t_{i}} B_{i}^{k}(x)+\bar{\alpha}_{i+1, j+1}^{k}(x),
\end{aligned}
$$

because $B_{j+1}^{k}(x)=0$ for $x \in\left[t_{j}, t_{j+1}\right)$. Thus we have proved the recurrence

$$
\bar{\alpha}_{i, j+1}^{k+1}(x)=\frac{x-t_{i}}{t_{i+k}-t_{i}} B_{i}^{k}(x)+\bar{\alpha}_{i+1, j+1}^{k}(x),
$$

for $x \in\left[t_{j}, t_{j+1}\right)$ and $j=i, \ldots, i+k-1$. We proceed to manipulate (5) to get a more symmetric expression. Obviously,

$$
\begin{aligned}
\bar{\alpha}_{i, j+1}^{k}(x) & =\sum_{r=i}^{j} B_{r}^{k}(x)=B_{i}^{k}(x)+\sum_{r=i+1}^{j} B_{r}^{k}(x) \\
& =B_{i}^{k}(x)+\bar{\alpha}_{i+1, j+1}^{k}(x),
\end{aligned}
$$


whence $B_{i}^{k}(x)=\bar{\alpha}_{i, j+1}^{k}(x)-\bar{\alpha}_{i+1, j+1}^{k}(x)$, which, when substituted in (5) gives

$$
\begin{aligned}
\bar{\alpha}_{i, j+1}^{k+1}(x) & =\frac{x-t_{i}}{t_{i+k}-t_{i}}\left(\bar{\alpha}_{i, j+1}^{k}(x)-\bar{\alpha}_{i+1, j+1}^{k}(x)\right)+\bar{\alpha}_{i+1, j+1}^{k}(x) \\
& =\frac{x-t_{i}}{t_{i+k}-t_{i}} \bar{\alpha}_{i, j+1}^{k}(x)+\bar{\alpha}_{i+1, j+1}^{k}(x)\left(1-\frac{x-t_{i}}{t_{i+k}-t_{i}}\right) .
\end{aligned}
$$

Finally, we have the recurrence

$$
\bar{\alpha}_{i, j+1}^{k+1}(x)=\frac{x-t_{i}}{t_{i+k}-t_{i}} \bar{\alpha}_{i, j+1}^{k}(x)+\frac{t_{i+k}-x}{t_{i+k}-t_{i}} \bar{\alpha}_{i+1, j+1}^{k}(x),
$$

for $x \in\left[t_{j}, t_{j+1}\right)$ and $j=i, \ldots, i+k-1$.

We need to evaluate

$$
\frac{1}{w_{j}} \frac{t_{i+k}-t_{i}}{k}\left(\sum_{r=i}^{j} B_{r}^{k+1}(x)-\sum_{r=i}^{j-1} B_{r}^{k+1}\left(t_{j}\right)\right)=\frac{t_{i+k}-t_{i}}{k w_{j}}\left(\bar{\alpha}_{i, j+1}^{k+1}(x)-\alpha_{i, j}^{k+1}\right),
$$

but have no way of telling whether the subtraction of $\bar{\alpha}$ 's will result in dangerous cancellation of significant digits; therefore we must find another way of evaluating differences of $\bar{\alpha}$ 's. To this end, let

$$
\bar{\delta}_{i, j}^{k+1}(x):=\bar{\alpha}_{i, j+1}^{k+1}(x)-\alpha_{i, j}^{k+1} .
$$

From (6) we have

$$
\begin{aligned}
& \bar{\delta}_{i, j}^{k+1}(x) \\
& =\frac{x-t_{i}}{t_{i+k}-t_{i}} \bar{\alpha}_{i, j+1}^{k}(x)+\frac{t_{i+k}-x}{t_{i+k}-t_{i}} \bar{\alpha}_{i+1, j+1}^{k}(x)-\frac{t_{j}-t_{i}}{t_{i+k}-t_{i}} \alpha_{i, j}^{k}-\frac{t_{i+k}-t_{j}}{t_{i+k}-t_{i}} \alpha_{i+1, j}^{k} \\
& =\frac{t_{j}-t_{i}}{t_{i+k}-t_{i}} \bar{\delta}_{i, j}^{k}(x)+\frac{t_{i+k}-x}{t_{i+k}-t_{i}} \bar{\delta}_{i+1, j}^{k}(x)+\frac{x-t_{j}}{t_{i+k}-t_{i}}\left(\bar{\alpha}_{i, j+1}^{k}(x)-\alpha_{i+1, j}^{k}\right) .
\end{aligned}
$$

Further,

$$
\begin{aligned}
\bar{\alpha}_{i, j+1}^{k}(x)-\alpha_{i+1, j}^{k} & =\bar{\alpha}_{i, j+1}^{k}(x)-\bar{\alpha}_{i+1, j+1}^{k}(x)+\bar{\alpha}_{i+1, j+1}^{k}(x)-\alpha_{i+1, j}^{k} \\
& =\bar{\alpha}_{i, j+1}^{k}(x)-\bar{\alpha}_{i+1, j+1}^{k}(x)+\bar{\delta}_{i+1, j}^{k}(x) \\
& =\sum_{r=i}^{j} B_{r}^{k}(x)-\sum_{r=i+1}^{j} B_{r}^{k}(x)+\bar{\delta}_{i+1, j}^{k}(x) \\
& =B_{i}^{k}(x)+\bar{\delta}_{i+1, j}^{k}(x)
\end{aligned}
$$

where the last line follows from the defining equation (3) for $\bar{\delta}_{i+1, j}^{k}(x)$. On substituting (8) in (7) we get

$$
\bar{\delta}_{i, j}^{k+1}(x)=\frac{t_{j}-t_{i}}{t_{i+k}-t_{i}} \bar{\delta}_{i, j}^{k}(x)+\frac{t_{i+k}-t_{j}}{t_{i+k}-t_{i}} \bar{\delta}_{i+1, j}^{k}(x)+\frac{x-t_{j}}{t_{i+k}-t_{i}} B_{i}^{k}(x),
$$

for $x \in\left[t_{j}, t_{j+1}\right)$ and $j=i, \ldots, i+k-1$. Finally, from (4) we have

$$
\frac{k}{t_{i+k}-t_{i}} \int_{t_{i}}^{x} B_{i}^{k}(\tau) \frac{d \tau}{w(\tau)}=\sum_{s=i}^{j-1} \frac{\delta_{i, s}^{k+1}}{w_{s}}+\frac{1}{w_{j}} \bar{\delta}_{i, j}^{k+1}(x)
$$


with

$$
\delta_{i, s}^{k+1}:=\bar{\delta}_{i, s}^{k+1}\left(t_{s+1}\right),
$$

$x \in\left[t_{j}, t_{j+1}\right)$ and $j=i, \ldots, i+k-1$. Specially,

$$
\frac{k}{t_{i+k}-t_{i}} \int_{t_{i}}^{t_{i+k}} B_{i}^{k}(\tau) \frac{d \tau}{w(\tau)}=\sum_{s=i}^{i+k-1} \frac{\delta_{i, s}^{k+1}}{w_{s}},
$$

and by (9)

$$
\frac{k}{t_{i+k}-t_{i}} \int_{t_{j}}^{t_{j+1}} B_{i}^{k}(\tau) d \tau=w_{j}\left(\int_{t_{i}}^{t_{j+1}} B_{i}^{k}(\tau) \frac{d \tau}{w(\tau)}-\int_{t_{i}}^{t_{j}} B_{i}^{k}(\tau) \frac{d \tau}{w(\tau)}\right)=\delta_{i, j}^{k+1},
$$

where $\delta_{i, j}^{k+1}$ is calculated recursively:

$$
\begin{aligned}
\delta_{i, j}^{2} & =\left\{\begin{array}{l}
1 \text { for } j=i, \\
0 \text { for } j \neq i,
\end{array}\right. \\
\delta_{i, j}^{k+1} & =\frac{t_{j}-t_{i}}{t_{i+k}-t_{i}} \delta_{i, j}^{k}+\frac{t_{i+k}-t_{j}}{t_{i+k}-t_{i}} \delta_{i+1, j}^{k}+\frac{t_{j+1}-t_{j}}{t_{i+k}-t_{i}} B_{i}^{k}\left(t_{j+1}\right),
\end{aligned}
$$

for $j=i, \ldots, i+k-1$.

\section{Conclusion}

There are other ways of calculating weighted integrals of polynomial splines, like Gaussian integration or conversion to Bezier form, and also some approximative ones [17]. In fact, (10) is a special case of recurrence used to evaluate inner products of B-splines ([4]) in which one of the B-splines is of order one. The proof given here is more in the spirit of 'B-splines without divided differences' [5], contains some new recurrences (5), and can be extended to obtain a recurrence for inner products. For inner products though, the greater complexity $\left(\mathrm{O}\left(k^{4}\right)\right)$ compared to Gaussian integration $\left(\mathrm{O}\left(k^{3}\right)\right)$ makes the recurrence seldom used, while for weighted splines it is preferable, being of the same complexity and machine independent.

\section{Acknowledgement}

This research was supported by grant 0037114, by the Ministry of Science, Education and Sports of the Republic of Croatia.

\section{References}

1. D. Bister and H. Prautzsch: A new approach to Tchebycheffian B-splines. In: Curves and Surfaces with Applications in CAGD, A. Le Méhauté, C. Rabut, L.L. Schumaker (eds.), Vanderbilt University Press, Nashville, 1997, 387-394.

2. C. de Boor: A Practical Guide to Splines. Springer, New York, 1978. 
3. C. de Boor: Calculation of the smoothing spline with weighted roughness measure. Math. Models Methods Appl. Sci. 11(1), 2001, 33-41.

4. C. de Boor, T. Lyche and L.L. Schumaker: On calculating with B-splines II: integration. In: Numerische Methoden der Approximationstheorie, L. Collatz, H. Werner and G. Meinardus (eds.), Birkhäuser, Basel, 1976, 123-146.

5. C. de Boor and K. Höllig: B-splines without divided differences. In: Geometric Modeling, G. Farin (ed.), SIAM, Philadelphia, 1987, 21-27.

6. L. Bos and K. Salkauskas: Weighted splines based on piecewise polynomial weight functions. In: Curve and Surface Design, H. Hagen (ed.), SIAM, Philadelphia, 1999, 87-98.

7. L. Bos and K. Salkauskas: Limits of weighted splines based on piecewise constant weight functions. Rocky Mountain J. Math. 23, 1993, 483-493.

8. T. Bosner: Knot insertion algorithms for weighted splines. In: Proceedings of the Conference on Applied Mathematics and Scientific Computing, Z. Drmač, M. Marušić and Z. Tutek (eds.), Springer, 2005, 151-160.

9. T.A. Foley: Interpolation with interval and point tension controls using cubic weighted $\nu$-splines. ACM Trans. Math. Software 13(1), 1987, 68-96.

10. T.A. Foley: Local control of interval tension using weighted splines. CAGD 3, 1986, 281-294.

11. T.A. Foley: Weighted bicubic spline interpolation to rapidly varying data. ACM Trans. on Graphics 6, 1987, 1-18.

12. M.-L. Mazure: Blossoming: a geometrical approach. Constr. Approx. 15, 1999, $33-68$.

13. S. Pruess: Alternatives to the exponential spline in tension. Math. Comp. 33, 1979, 1273-1281.

14. M. Rogina: A knot insertion algorithm for weighted cubic splines. In: Curves and Surfaces with Applications in CAGD, A. Le Méhauté, C. Rabut, L.L. Schumaker (eds.), Vanderbilt University Press, Nashville, 1997, 387-394.

15. M. Rogina: Algebraic proof of the B-spline derivative formula. In: Proceedings of the Conference on Applied Mathematics and Scientific Computing, Z. Drmač, M. Marušić, Z. Tutek (eds.), Springer, 2005, 273-281.

16. L.L. Schumaker: Spline Functions: Basic Theory. John Wiley \& Sons, New York, 1981.

17. A.H. Vermeulen, R.H. Bartels, and G.R. Heppler: Integrating products of Bsplines. SIAM J. Sci. Stat. Comput. 13(4), 1992, 1025-1038. 